\title{
Grupos de pesquisa em educação em Enfermagem: linhas de pesquisa e produção científica em três regiões do Brasil
}

\author{
Research groups in nursing education: \\ research areas and scientific production in three regions of Brazil \\ Grupos de investigación en educación en enfermería: \\ líneas de investigación y producción científica en tres regiones del Brasil
}

\begin{abstract}
Mariana Cabral Schveitzer', Vânia Marli Schubert Backes", Marta Lenise do Prado", Mônica Motta Lino"', Fabiane Ferraz"

' Universidade de São Paulo, Escola de Enfermagem, Programa de Pós-Graduação em Enfermagem. (Doutoranda) São Paulo-SP, Brasil.

"Universidade Federal de Santa Catarina, Departamento de Enfermagem, Programa de Pós-Graduação em Enfermagem, Grupo de Pesquisa em Educação em Enfermagem e Saúde. Florianópolis-SC, Brasil.

II' Universidade Federal de Santa Catarina, Programa de Pós-Graduação em Enfermagem, Grupo de Pesquisa em Educação em Enfermagem e Saúde. (Doutoranda) Florianópolis-SC, Brasil.
\end{abstract}

Submissão: 09-02-2011 Aprovação: 23-04-2012

\section{RESUMO}

Este estudo objetivou identificar as temáticas das linhas de pesquisa (LP) e da produção científica publicada pelos Grupos de Pesquisa em Educação em Enfermagem (GPEE) das regiões Norte, Nordeste e Centro-Oeste do Brasil. Pesquisa documental, descritiva e exploratória. Identificaram-se 12 GPEE, 45 linhas de pesquisa e 448 artigos científicos, $60 \%$ dos quais foram publicados em revistas A, B1 e B2. Nesses GPEE, 36\% das LP foram sobre educação, porém somente $25 \%$ das publicações abordaram este assunto, enquanto $34 \%$ das LP enfocaram o tema cuidado, que foi objeto de $44 \%$ das publicações. Há necessidade de repensar a organização dos GPEE a partir de linhas e projetos de pesquisa para favorecer a consolidação da temática da Educação em Enfermagem na produção de conhecimentos da área.

Descritores: Educação em enfermagem; Pesquisa em enfermagem; Pesquisa em educação em enfermagem.

ABSTRACT
This study aimed to identify themes of research areas and scientific literature of Research Groups in Nursing Education (GPEE in Portuguese) of the North, Northeast and Midwest regions of Brazil. This is a descriptive and exploratory study. It was identified 12 GPEE, 45 fields of research and 448 scientific papers, of which $60 \%$ have appeared in magazines classified as A, B1 and B2. Even though $36 \%$ of the research areas were about education, only $25 \%$ of the papers covered this theme. Furthermore, $34 \%$ of the research areas were about care and $44 \%$ of the papers were about it. It's necessary to rethink the GPEE organization in regards to areas and research projects, to advance towards the consolidation of Nursing Education as an area of knowledge production.

Key words: Nursing education; Nursing research; Research in nursing education.

\section{RESUMEN}

El estudio objetivó identificar los temas de las líneas de investigación (LI) y de la producción científica desarrollada por los Grupos de Investigación en Educación en Enfermería (GPEE) del Norte, Nordeste y Centro-Oeste de Brasil. Investigación documental, descriptiva y exploratoria. Se identificaron 12 GPEE, 45 LI y 448 artículos científicos, 60\% fueran publicados en revistas A, B1, B2. En los GPEE, 36\% de las LI fueran sobre educación, pero solamente $25 \%$ de las publicaciones abordaran este asunto. Todavía 34\% de las LI presentaran el tema cuidado, y 44\% de las publicaciones fueran sobre este asunto. Es necesario repensar la organización de los GPEE, líneas y proyectos de investigación, para avanzar en la consolidación de la Educación en Enfermería como productora de conocimiento.

Palabras clave: Educación en enfermería; Investigación en enfermería; Investigación en la educación de enfermería.

\section{AUTOR CORRESPONDENTE Mariana Cabral Schveitzer E-mail: marycabral101@gmail.com}




\section{INTRODUÇÃO}

O avanço científico de uma determinada área depende de inúmeros fatores. Na área da saúde, em especial na Enfermagem, destacam-se a capacidade de produzir conhecimentos novos a partir da competência das instituições de ensino, principalmente em nível de pós-graduação, em formar investigadores e do interesse dos profissionais em buscar capacitação por meio de programas de mestrado e doutorado, para desenvolver pesquisa a partir das necessidades dos distintos setores da saúde. Também se faz necessário viabilizar políticas e prioridades em pesquisas, por meio das quais seja possível apoiar Grupos de Pesquisa (GP) e, por conseguinte, fortalecer linhas de investigação a partir das prioridades atribuídas para as diferentes áreas de um país(1-3).

$\mathrm{Na}$ Enfermagem brasileira, o processo de desenvolvimento científico estruturou-se a partir da década de 1970, com a instituição de cursos de pós-graduação stricto sensu e o estabelecimento dos Grupos de Pesquisa em algumas universidades públicas. Os Grupos de Pesquisa foram criados para atender as demandas dos programas de mestrado e doutorado, que precisavam de espaços para discutir os projetos de pesquisa e seus resultados entre professores, estudantes de graduação e pós-graduação e pessoal de apoio técnico ${ }^{(1,4-5)}$.

Segundo o Conselho Nacional de Desenvolvimento Científico e Tecnológico (CNPq), os Grupos de Pesquisa organizam-se em torno de linhas de pesquisa segundo uma regra hierárquica fundada na experiência e na competência técnico-científica dos pesquisadores. As linhas de pesquisa representam temas aglutinadores de estudos científicos que se fundamentam em tradição investigativa, de onde se originam projetos cujos resultados guardam afinidades entre si. Dessa forma, as linhas de pesquisa subordinam-se ao grupo, e não o contrário ${ }^{(4)}$.

Assim entende-se que as linhas de pesquisa podem sofrer alterações, pois dependem dos participantes dos GP. Atualmente o CNPq considera atípica a participação de um pesquisador em mais de quatro Grupos de Pesquisa e alguns Programas de Pós-Graduação exigem que as propostas de pesquisa submetidas ao processo seletivo dos cursos de mestrado e doutorado estejam de acordo com as linhas dos Grupos de Pesquisa do Programa ${ }^{(4)}$.

Essas medidas decorrem do fato que a adoção de linhas de pesquisa pelos GP pode facilitar a definição dos projetos de pesquisa e gerar maior afinidade entre os estudos realizados pelos membros do Grupo. Desse modo, "cada vez mais tem se buscado uma maior interface entre as temáticas nas linhas de pesquisa dentro dos grupos, de modo a consolidar a construção de conhecimento de forma mais gregária, menos segmentada, numa perspectiva de complementaridade dos projetos de pesquisa desenvolvidos e seus resultados"(6:4).

Frente a isso, como avaliar se os GP estão realmente desenvolvendo suas investigações dentro das linhas de pesquisa propostas? Segundo o Censo CNPq de 2008, existem no Brasil trezentos e setenta e três (373) Grupos de Pesquisa em Enfermagem e um mil e cinquenta e nove (1.059) linhas de pesquisa, determinando que, em média, cada GP se organiza a partir de duas ou três linhas de pesquisa ${ }^{(4)}$.
Desde 2007 o Grupo de Pesquisas em Educação em Enfermagem e Saúde (EDEN), do Programa de Pós-Graduação em Enfermagem da Universidade Federal de Santa Catarina (UFSC), tem participado ativamente de um projeto de pesquisa multicêntrico intitulado "A produção investigativa de Educação em Enfermagem: o estado da arte" resultado de uma parceria entre pesquisadores da Universidade Nacional de Colômbia da Faculdade de Enfermagem e pesquisadores brasileiros, convidados por meio da Associação Brasileira de Enfermagem (ABEn), a partir de uma necessidade de expandir o estudo para o âmbito ibero-americano. Desta forma, ampliou-se para o cenário brasileiro o questionamento sobre as diferentes publicações, resultantes de processos investigativos, tendências metodológicas, reflexões e conclusões formuladas por pesquisadores de Educação em Enfermagem, analisando os dados por Regiões Geográficas do país.

No processo de análise da Região Sul do Brasil já se alcançaram achados importantes no tocante a caracterização dos Grupos de Pesquisa em Educação em Enfermagem (GPEE), nos quais cumpre destacar: a baixa interdisciplinaridade na composição destes Grupos; a alta qualificação dos pesquisadores, tendo em vista que $86 \%$ possuem a titulação de mestrado e doutorado; a baixa presença de estudantes de graduação nestes espaços; a necessidade da criação de uma política de integração entre o ensino, o serviço e a pesquisa; o escasso fomento para o desenvolvimento das pesquisas que são desenvolvidas; e, a importância da integração de interesses para a criação de redes colaborativas no setor, que estimule o espírito científico, o pensamento crítico-reflexivo e o consequente fortalecimento da profissão(2).

$\mathrm{Na}$ Região Sudeste, existe a concentração de financiamento e centros de excelência tanto no setor de pesquisa em saúde quanto na distribuição dos recursos humanos, revelando iniquidades regionais quando comparado com as regiões Norte, Nordeste e Centro-Oeste do Brasil ${ }^{(7)}$. Assim, está discutido os esforços que estão sendo empregados a partir da década de 1990 para fixar doutores nas universidades em outras regiões brasileiras, promovendo uma possível descentralização da pesquisa em saúde, porém, ainda hoje o número de programas de graduação e pós-graduação em enfermagem é menor nas Regiões Norte, Nordeste e Centro-Oeste quando comparados à Região Sudeste ${ }^{(3,6-6)}$.

Como uma das atribuições do GP para avançar no processo de desenvolvimento científico é produzir novos conhecimentos a partir das linhas de pesquisa que o compõem, bem como socializar estes resultados, poderia a produção científica publicada pelos pesquisadores de um GP demonstrar essa congruência?

Com a intenção de responder esta pergunta, o presente estudo tem por objetivo identificar as temáticas das linhas de pesquisa (LP) e da produção científica publicada pelos Grupos de Pesquisa em Educação em Enfermagem (GPEE) de três regiões do Brasil.

\section{MÉTODO}

Trata-se de um estudo documental, exploratório, descritivo, com adoção da Análise de Conteúdo ${ }^{(7)}$. Para a coleta dos 
dados, desenvolveram-se os seguintes passos: acesso ao site do CNPq (<www.cnpq.br >,) selecionando "Banco de Dados e Estatísticas" e, em seguida, "Grupos de Pesquisa - Censos" e, finalmente, "Plano Tabular". Escolhido o ano do censo, neste caso 2008, foram selecionadas as seguintes variáveis: "Área de Atuação", "Por UF", "Por Instituição". Na sequência, foi realizado o filtro da primeira variável, sendo escolhida a área "Enfermagem". Assim, o sistema gerou uma tabela em que se realizou a coleta dos dados discriminados a partir das variáveis selecionadas do total de Grupos de Pesquisa em Enfermagem no Brasil em 2008. Foram construídas tabelas no Microsoft Excel $2003^{\circledR}$ divididas por região geográfica.

Para cada uma das regiões Norte (N), Nordeste (NE) e Centro-Oeste $(\mathrm{CO})$ foram identificados os seguintes elementos: instituições e caráter institucional das mesmas; número total de Grupos de Pesquisa na área de Enfermagem; número específico de Grupo de Pesquisa em Educação em Enfermagem (GPEE), sendo que o critério para identificação foi apresentar a palavra "educação" ou sinônimos (ensino e formação) no nome do grupo; número total de Linhas de Pesquisa; presença de Programas de Pós-Graduação stricto sensu em Enfermagem; nome completo, ano de início e a sigla do GPEE; número, formação, titulação e atuação profissional dos pesquisadores e técnicos dos GPEE; número, formação e titulação dos estudantes dos GPEE; número de Bolsistas de Iniciação Científica entre os estudantes de graduação.

Neste artigo são apresentados as seguintes variáveis: número de Grupo de Pesquisa em Educação em Enfermagem (GPEE) das três regiões estudadas, número de Linhas de Pesquisa por GPEE e produção científica dos pesquisadores que atuam nos GPEE. Em relação à produção científica os dados foram complementados por meio das informações disponibilizadas on-line no Diretório dos Grupos de Pesquisa do CNPq, no currículo Lattes dos pesquisadores e no site da Coordenação de Aperfeiçoamento de Pessoal de Nível Superior (CAPES).

Além da distribuição quantitativa da produção de artigos científicos, também foi verificada a qualificação dos periódicos em que foram publicados, por meio da lista de veículo de divulgação científica denominada Qualis/CAPES referente ao ano de
2008. Segundo esse indicador, a produção intelectual dos programas de Pós-Graduação strictu sensu é realizada pelas áreas de avaliação e passa por processo anual de atualização e esses veículos de divulgação científica são enquadrados em estratos indicativos da qualidade, sendo $\mathrm{A} 1 \mathrm{o}$ nível mais elevado, seguido por A2; B1; B2; B3; B4; B5 e C, este com peso zero. O aplicativo que permite a classificação e consulta ao Qualis das áreas, bem como a divulgação dos critérios utilizados para a classificação de periódicos, é o WebQualis, disponível no portal virtual da CAPES - http://qualis.capes.gov.br/webqualis.

As linhas de pesquisa citadas pelos GPEE que constam do Diretório do $\mathrm{CNPq}$ foram dispostas em um quadro e organizadas por convergência de tema em cinco grandes áreas, a saber: educação; cuidado; gestão e trabalho; ética; e saúde mental. Entre os artigos científicos publicados no período de 2004-2008 e citados no currículo Lattes dos pesquisadores dos GPEE, os que estavam disponibilizados on-line foram captados na íntegra e organizados no gerenciador bibliográfico EndNote ${ }^{\circledast}$. O Quadro 1 exemplifica como foi realizada a categorização das linhas de pesquisa de um GPEE da região Centro-oeste, com oito linhas de pesquisa.

A leitura dos resumos dos artigos permitiu classificar a produção científica dos grupos em cinco temas: Educação em Enfermagem e em Saúde, Cuidado em Enfermagem e Saúde, Estudos Epidemiológicos, Processo de Trabalho em Saúde e Outros temas.

Vale ressaltar que essas categorizações foram embasadas na Análise de Conteúdo, que permite "classificar elementos em categorias impõe a investigação do que cada um deles tem em comum com outros. O que vai permitir o seu agrupamento é a parte comum existente entre eles. É possível, contudo, que outros critérios insistam noutros aspectos de analogia, talvez modificando consideravelmente a repartição anterior" (8:112).

Como se tratar de uma pesquisa documental em que as informações estão disponibilizadas em bases de dados de domínio público, o estudo não foi submetido ao Comitê de Ética em Pesquisa com Seres Humanos. No entanto, cabe ressaltar que foram seguidos os preceitos éticos para o tratamento dos dados em pesquisas dessa natureza.

\begin{tabular}{|l|l|}
\multicolumn{1}{|c|}{ Linha de Pesquisa } & \multicolumn{1}{|c|}{ Categoria } \\
\hline $\begin{array}{l}\text { Abordagens Metodológicas e epistemológicas do conceito de curar/cuidar. } \\
\text { Determinantes do Processo Saúde-Doença } \\
\text { Fundamentos do Processo de Cuidar, Tecnologia e Instrumentação }\end{array}$ & 3 Cuidado \\
\hline Bioética & 1 Ética \\
\hline $\begin{array}{l}\text { Depressão em crianças e adolescentes } \\
\text { Psiquiatria da criança e do adolescente }\end{array}$ & 2 Saúde mental \\
\hline Educação, Saúde, Sociedade e Tecnopolos & 1Educação \\
\hline Gestão da atenção e organização dos serviços de saúde e enfermagem & 1 Gestão e trabalho \\
\hline
\end{tabular}

Quadro 1 - Linhas de pesquisa do GPEE da região Centro-Oeste. CNPq, 2010. 
Tabela 1 - GPEE e respectivas linhas de pesquisa categorizadas nas cinco áreas. CNPq, 2010.

Sigla Região/Sigla Instituição/Ano de formação e nome do GPEE

CO/UNB/1992 - Núcleo de Estudos em Educação, Promoção em Saúde e Projetos Inclusivos

CO/UFMS/2002 - Educação em Saúde

CO/UNB/2003 - Núcleo de Estudos em Enfermagem, Educação,

Processo de Trabalho em Saúde, Ambiente e Vigilância

CO/UFMT/2008 - Educação, Formação e Gestão em Saúde e em Enfermagem

NE/UFC/1993 - Família, Ensino, Pesquisa e Extensão

NE/UFBA/2000 - Educação, Ética e Exercício da Enfermagem

NE/UFMA/2005 - Núcleo de Estudos e Pesquisa de Enfermagem em Educação e Saúde da Mulher

NE/UFPI/2005 - Núcleo de Estudos e Pesquisas em Trabalho e Educação na Saúde

NE/UERN/2007 - O processo de trabalho na saúde, a especificidade do trabalho do enfermeiro e a Formação de Recursos Humanos e Educação em Saúde

NE/UFRN/2007 - Educação em Enfermagem

NE/UFPI/2008 - Educação e História em Enfermagem e Saúde

N/UFPA/2005 - Educação, Política e Tecnologia em Enfermagem da Amazônia

\section{Categoria das Linhas de Pesquisa}

3 Cuidado, 1 ética, 2 saúde mental, 1 Educação, 1 gestão e trabalho

\section{Educação}

2 Cuidado, 3 gestão e trabalho

1 Educação, 1 gestão e trabalho

1 Educação

2 Cuidado, 3 ética, 1 educação

3 Cuidado, 1 educação, 1 ética

1 Gestão e trabalho, 1 educação,

4 Cuidado, 2 educação

4 Educação, 1 ética

1 Educação

1 Educação, 1 cuidado

\section{RESULTADOS}

Foram identificados doze (12) GPEE nas Regiões Norte, Nordeste e Centro-Oeste do Brasil que apresentaram quarenta e cinco (45) linhas de pesquisa. A Tabela reúne os dados que relacionam os GPEE segundo o número de LP e suas respectivas temáticas expressas nas cinco categorias já mencionadas.

Verificou-se que dezesseis (16) linhas de pesquisa possuíam relação com o tema educação, como currículo, práticas educativas, ensino e formação profissional; quinze (15) relacionavam-se ao tema cuidado, como atenção a saúde da mulher e assistência de enfermagem; sete (07), com o tema gestão e trabalho, por exemplo, gestão de pessoas e organização dos serviços de saúde; cinco (05), com o tema ética - que incluía bioética e questões de cidadania e duas (02), com o tema saúde mental, como depressão e problemas psiquiátricos em crianças e adolescentes.

No total foram encontrados quatrocentos e quarenta e oito (448) artigos científicos entre os anos de 2004-2008, sendo que duzentos e sessenta e sete (267) foram publicados em revistas A, B1 e B2, de acordo com o Qualis/CAPES de 2008. Duzentos e vinte e nove (229) foram acessados on-line na íntegra e tornaram-se objeto de análise desta investigação. Esses artigos foram categorizados em cinco grandes temas de interesse: Educação em Enfermagem e em Saúde, Cuidado em Enfermagem e Saúde, Estudos Epidemiológicos, Processo de Trabalho em Saúde e Outros temas. Os resultados são apresentados a seguir, divididos por Região Geográfica.

\section{Região Norte}

O único GPEE da Região Norte encontra-se no estado do Pará. Apresenta duas linhas de pesquisa, uma sobre educação e outra sobre cuidado. Publicou onze (11) artigos científicos entre os anos de 2004-2008, sendo que oito (8) foram publicados em revistas A1, A2, B1 e B2, que classificados a partir de temas de interesse na tabela 2 .

Tabela 2 - Classificação da produção de artigos científicos disponíveis on-line, do Grupo de Pesquisa em Educação em Enfermagem da Região Norte do Brasil, no período de 20042008, segundo temas de interesse.

\begin{tabular}{lc}
\hline & \multicolumn{1}{c}{$\begin{array}{c}\text { Unidade da Federação } \\
\text { Brasileira }\end{array}$} \\
\cline { 2 - 2 } Temas de Interesse & PA \\
\hline Educação em Enfermagem e em Saúde & 3 \\
Cuidado em Enfermagem e Saúde & 2 \\
Estudos Epidemiológicos & 2 \\
Outros temas* & 1 \\
Total & $\mathbf{8}$ \\
\hline
\end{tabular}

*Alcoolismo.

\section{Região Nordeste}

Na região Nordeste, existem sete (7) GPEE distribuídos em cinco (5) Estados, com um total de vinte e seis (26) linhas de 
Tabela 3 - Classificação da produção de artigos científicos disponíveis on-line, dos Grupos de Pesquisa em Educação em Enfermagem da Região Nordeste do Brasil, no período de 2004-2008, segundo temas de interesse.

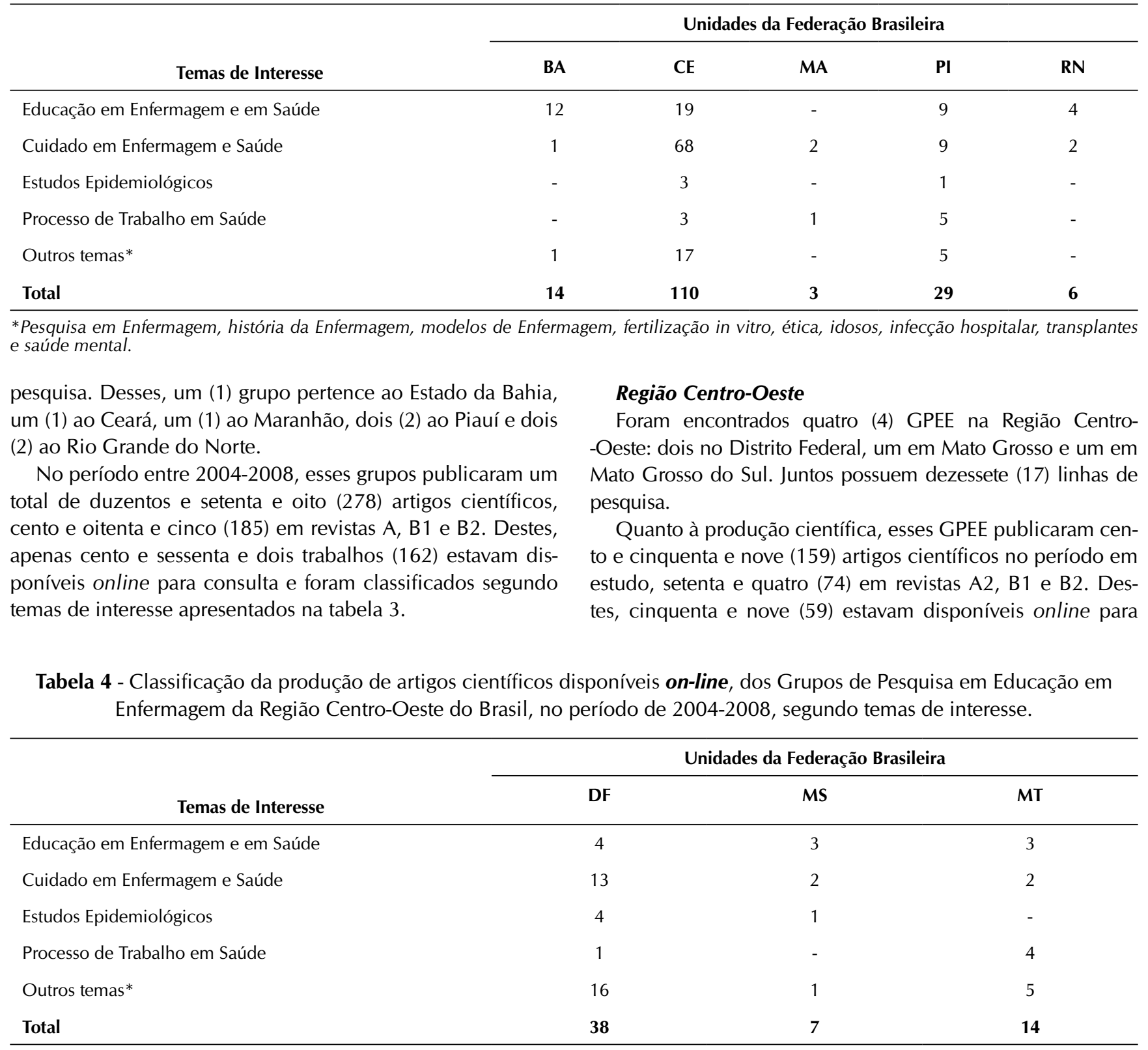

*Saúde mental, trabalho geral (ambiente organizacional, treinamento, aprendizagem), idoso e Paidéia (família e escola).

Tabela 5 - Classificação da produção dos artigos científicos disponíveis on-line, dos Grupos de Pesquisa em Educação em Enfermagem das Regiões Norte, Nordeste e Centro-Oeste do Brasil, no período de 2004-2008, segundo temas de interesse.

\begin{tabular}{lc}
\hline \multicolumn{1}{c}{ Temas de interesse } & Regiões N, NE e CO \\
\hline Cuidado em Enfermagem e em Saúde & 101 \\
Educação em Enfermagem e em Saúde & 57 \\
Estudos Epidemiológicos & 11 \\
Processo de Trabalho em Saúde & 14 \\
Outros temas & 46 \\
Total & $\mathbf{2 2 9}$ \\
\hline
\end{tabular}


consulta e foram classificados segundo temas de interesse, conforme a tabela 4.

A produção científica dos 12 GPEE das Regiões Norte, Nordeste e Centro-Oeste, agrupada por temas de interesse, revela o panorama expresso na tabela 5 .

Vale ressaltar que dentre os cento e vinte e três (123) artigos publicados em revistas B3, B4 ou B5 pelos GPEE, entre os diversos temas abordados, também foram encontrados estudos que discutiam o tema educação, a saber: práticas educativas, educação em saúde, políticas públicas e formação do enfermeiro.

\section{DISCUSSÃO}

O número de linhas de pesquisa por GPEE das Regiões Norte, Nordeste e Centro-Oeste corresponde à média brasileira de Grupos de Pesquisa em Enfermagem, de três linhas de pesquisa por Grupo $^{(9)}$. Todavia este dado não pode ser generalizado, pois foram encontrados grupos com uma única linha de pesquisa e outros com até oito linhas. Um estudo sobre grupos de pesquisa em Enfermagem realizado em 2009 constatou que $92 \%$ dos grupos possuíam entre 1 a 5 linhas de pesquisa, organização que permite um maior direcionamento da produção do conhecimento às linhas de pesquisa dos grupos, sistematizando a produção científica ${ }^{(9)}$.

Dentre os GPEE selecionados para este estudo, um não possuía LP em educação e apenas três apresentaram LP somente sobre educação. Visto que são GPEE, vale refletir sobre o papel da LP nessa organização; o fato de ter mais de uma linha pode prejudicar o desenvolvimento das pesquisas na área específica de educação e, consequentemente, a produção científica do grupo?

Ao definir suas LP, um grupo de pesquisa o faz no sentido de organizar um guia no qual irá estruturar seus estudos e suas pesquisas. Um GPEE deve ser reconhecido pelo seu papel na pesquisa sobre educação em enfermagem. Atualmente, a forma mais difundida um grupo de pesquisa tornar-se conhecido no meio acadêmico é por meio da publicação de artigos científi$\cos ^{(5)}$, não somente pela quantidade, mas pela qualidade desta produção. Sendo assim, anualmente são avaliados o nível dos periódicos e seu impacto em nível nacional (revistas conceito B2, B3, B4, B5) e internacional (revistas conceito A e B1) ${ }^{(4)}$.

Em relação à relevância da produção científica, vale destacar que $60 \%$ dos artigos científicos foram publicados em revistas conceito A, B1 e B2, de acordo com o Qualis/CAPES de 2008. Esse dado é bastante promissor e indica o esforço dos GPEE no investimento na publicação em revistas de impacto nacional, mas principalmente internacional, buscando a visibilidade e qualidade de sua produção.

Há entre os grupos de pesquisa os que defendem a solidariedade intelectual entre os pesquisadores e a superação de práticas endogênicas de produção, divulgação e consumo do conhecimento. Ademais, um espaço que permita a reunião de diferentes sujeitos - professores, alunos (graduação e pós-graduação), profissionais da assistência - para pesquisar e discutir um mesmo tema de interesse, pode reduzir a distância entre os campos de formação acadêmica/produção científica e a prática assistencial ${ }^{(10)}$.

Não parece ser esse o caso dos GPEE das Regiões Norte,
Nordeste e Centro-Oeste, nos quais somente $36 \%$ das linhas de pesquisa versavam sobre educação, temática que era abordada somente em 25\% das publicações. Contraditoriamente, $34 \%$ das linhas de pesquisa desses GPEE eram sobre cuidado, tema presente em $44 \%$ das publicações.

As LP são caminhos a serem seguidos pelos investigadores, o que ainda não se evidencia nos GPEE, pois os dados apontam que há mais publicações sobre cuidado que sobre educação nesses grupos. Essa indicação vale também para os demais Grupos de Pesquisa em Enfermagem no Brasil, visto que, em estudo publicado em 2009, as pesquisadoras encontraram que a indicação do vínculo das produções em linhas de pesquisa ainda mostra pouca clareza ${ }^{(9)}$. No caso dos GPEE, faz-se necessário instigar uma maior relação entre as LP apresentadas e a produção científica publicada no seu foco específico: a educação em enfermagem.

Outro fato a destacar nas publicações dos GPEE diz respeito ao item Outros Temas, pois esses artigos representaram 21\% das publicações, o que representa parcela considerável da produção científica dos GPEE e demonstra a necessidade de consolidação da produção científica nos temas e áreas definidos pelos próprios grupos, e em especial para o foco educativo.

A falta de vínculo entre a produção científica e as linhas de pesquisa pode ser devida à superposição de linhas, imbricação ou interfaces, concentração ou convergência, pulverização ou diversidade de temas, entre outros. Todavia, conforme o CNPq apregoa, é das linhas de pesquisa que deveriam surgir ou derivar os projetos dos GP nas suas diferentes modalidades ${ }^{(4,9)}$. Denota-se, portanto, a necessidade dos GPEE congregar a produção científica nas linhas de pesquisa, visto que os artigos científicos tendem a apresentar os resultados dos projetos de pesquisa elaborados e desenvolvidos a partir dessas linhas.

No caso dos GPEE das Regiões Norte, Nordeste, Centro-Oeste e Sul, essa congruência não foi observada, o que sugere a necessidade de alteração nas linhas de pesquisa dos Grupos ou uma revisão dos projetos de pesquisa. Esse processo deve ser acompanhado pela revisão reflexão dos membros do GP, pois a produção intelectual do pesquisador/docente deve compor linhas de pesquisa ${ }^{(9)}$. Dessa forma, é fundamental refletir sobre o significado das linhas de pesquisa de um grupo e sua implicação na produção de conhecimento.

A escolha de uma LP tem o objetivo de delimitar o foco das pesquisas desenvolvidas por um grupo que produz conhecimento substantivo sobre determinado assunto ${ }^{(9)}$. No caso dos GPEE, o foco é a educação em enfermagem e saúde. Esse fato deve ser levado em conta quando se considera a possibilidade de transformar a pesquisa em ato educativo, capaz de despertar o interesse e a co-responsabilidade dos envolvidos, enquanto sujeitos de um processo em construção, e converter a instituição de ensino em um cenário de intercâmbio reflexivo, produtor de conhecimentos ${ }^{(11,12)}$.

Os GPEE poderão demonstrar seu compromisso com a ciência ao produzir e publicar resultados a partir de pesquisas cientificamente orientadas, que integrem diferentes níveis de formação em um objetivo comum de avanço do conhecimento, o que possibilitará ampliar e desenvolver a capacidade de produção científica multidisciplinar, oferecendo 
maior visibilidade à Enfermagem ${ }^{(13)}$.

Em relação aos temas dos trabalhos sobre educação publicados em revistas conceito B3, B4 e B5, não foi encontrada diferença em relação aos temas sobre educação publicados nas revistas conceito A, B1 e B2. Isso leva a supor que a escoIha por esses veículos de divulgação não tenha ocorrido pela dificuldade de se publicar sobre o tema Educação em Enfermagem em periódicos indexados, mas sim devido à qualidade do material escrito ou ao público-alvo desejado.

\section{CONCLUSÕES}

Muitos arranjos e mudanças ainda são necessários para avançar na consolidação da Enfermagem brasileira como produtora de conhecimentos na área de educação em enfermagem, sendo que a possibilidade de refletir e repensar a organização dos Grupos de Pesquisa em Educação em Enfermagem, a partir de linhas e projetos de pesquisa, pode acelerar esse processo nessa área da Enfermagem.

Outros estudos são necessários para identificar se os resultados deste trabalho representam a realidade dos demais GPEE do Brasil e também de outros Grupos que abordam os diversos temas de pesquisa em Enfermagem. Nesse sentido, vale o desafio, tendo em vista a importância de repensar continuamente as práticas em saúde e Enfermagem, balizadas por conhecimentos científicos que são produzidos por meio de investigações, em sua maioria, desenvolvidas no interior dos Grupos de Pesquisa.

\section{REFERÊNCIAS}

1. Castrillón MC. Trends and Priorities in Nursing Research. Rev Latinoam Enferm 2004; 12(4):853-8.

2. Backes VMS, Canever BP, Ferraz F, Lino MM, Prado ML, Reibnitz KS. Grupos de Pesquisa em Educação em Enfermagem da Região Sul do Brasil. Rev Gaúch Enferm 2009;30(2):249-56.

3. Lino MM, Backes VMS, Canever BP, Ferraz F, Prado ML. Perfil da produção científica e tecnológica dos grupos de pesquisa em educação em enfermagem da Região Sul do Brasil. Rev Latinoam Enferm 2010;18(3):452-8.

4. Ministério de Ciências e Tecnologia [homepage na internet] Grupos de Pesquisa - Censo. [acesso em 01 abril 2010]. Disponível em: http://dgp.cnpq.br/censos/ inf gerais/index que eh.htm

5. Carvalho EC. A produção do conhecimento em enfermagem. Revista Latinoam Enferm 1998; 6(1):119-22.

6. Erdmann AL, Mello ALSF, Andrade SR, Koerich MS, Klock $P$, Nascimento KC. Sistema de Cuidados em Enfermagem e Saúde: As Interações Vivenciadas nos Grupos de Pesquisa. Ciênc Cuid Saúde 2009;8(4):675-82.

7. Gomes DC, Backes VMS, Lino MM, Canever BP, Ferraz
F, Schveitzer MC. Produção científica em Educação em Enfermagem: grupos de pesquisa Rio de Janeiro e Minas Gerais. Rev Gaúch Enferm 2011;32(2):330-7.

8. Bardin L. Análise de Conteúdo. Lisboa: Edições 70; 2007.

9. Erdmann AL, Lanzoni GMM. Características dos grupos de pesquisa da enfermagem brasileira certificados pelo CNPq de 2005 a 2007. Esc Anna Nery Rev Enferm 2008;12(2):316-22.

10. Cabral IE, Tyrrel, MAR Pesquisa em enfermagem nas Américas. Rev Bras Enferm 2010; 63(1):104-10.

11. Reibnitz KS, Prado ML. Formação do profissional crítico-criativo: a investigação como atitude de (re)conhecimento do mundo. Texto \& Contexto Enferm 2003;12(1):26-33

12. Krahl M, Sobiesiak EF, Poletto DS, Casarin RG, Knopf LA, Carvalho J, et al. Experiência dos acadêmicos de enfermagem em um grupo de pesquisa. Rev Bras Enferm 2009;62(1):146-50.

13. Barbosa SFF, Sasso GTM, Berns I. Enfermagem e Tecnologia: Análise dos Grupos de Pesquisa cadastrados na Plataforma Lattes do CNPQ. Texto \& Contexto Enferm 2009;18(3):443-8. 